\title{
PROGNOSTIC BIOMARKERS AND FACTORS IN POSTERIOR URETHRAL VALVES
}

\author{
Teodora Luchița ${ }^{1}$, Andreea Mădălina Șerban' ${ }^{1}$, Nicolae Sebastian Ionescu ${ }^{1,2}$
}

\section{Abstract}

Posterior urethral valves (PUV) are among the most common causes of obstructive nephropathies in the pediatric population. Despite early urinary drainage and endoscopic valve ablation, the evolution of this pathology is frequently oriented towards chronic kidney disease (CKD). Progressive renal failure is initiated by the intrauterine urethral obstruction, which in turn leads to the aggravation of the bladder dysfunction, even after the relief of the obstruction. These children need proper monitorization and management, based on their risk factors, in order to prevent or to delay the evolution to end stage renal disease. Early prognostic markers for renal failure are searched. So far, nadir creatinine and creatinine velocity proved useful in risk stratification. Other factors, biologic, imagistic and urodynamic, were found, but they still need to prove their degree of correlation on long term with renal function.

Keywords: posterior urethral valves, chronic kidney disease, bladder dysfunction, children

\section{Introduction}

Posterior urethral valves (PUV) are the most common cause of bladder outlet obstruction in male patients, with an incidence in the general population ranging between 1:70001:8000 [1]. The obstruction is represented by a fine

\section{Table 1. Prognostic factors for CKD evolution in patients with PUV}

\begin{tabular}{|c|c|}
\hline Antenatal factors & $\begin{array}{l}\text {-progressive oligohydramnios/ anhydramnios } \\
\text {-poor renal cortex echogenity } \\
\text { - poor bladder refilling }\end{array}$ \\
\hline Biologic markers & $\begin{array}{l}\text { - serum creatinine } \\
\text { - nadir creatinine } \\
\text { - creatinine velocity } \\
\text { - urinary cytokines TGF-1, TNF- } \alpha, \text { IL- } 6 \\
\text { - microalbuminuria } \\
\text { - plasmatic renin }\end{array}$ \\
\hline Imagistic measurements & $\begin{array}{l}\text {-SWRD index (cystography) } \\
\text { - estimated renal parenchyma } \\
\text { - the cortico-medullar index } \\
\text { - the renal echogenity }\end{array}$ \\
\hline Urodynamic studies & - the bladder contractility index \\
\hline
\end{tabular}

Adapted from Wein, A. J., Kavoussi, L. R., \& Campbell, M. F. (2012). Campbell-Walsh urology (10th ed.). Philadelphia: Elsevier Saunders, pp. 3252-3271.

1"Carol Davila" University of Medicine and Pharmacy, Bucharest

2"M.S. Curie" Clinical Emergency Hospital for Children, Bucharest. "Carol Davila" University of Medicine and Pharmacy, Bucharest

E-mail: teodora.luchita@gmail.com, serban.andreea.madalina@gmail.com,nsionescu@yahoo.com 
Antenatal diagnosis and prognostic factors

As for many other complex congenital conditions, the antenatal suspicion of posterior urethral valves is of paramount importance in planning the birth and placing the newborn in a tertiary center. Therefore, it is essential to identify the key elements of diagnosis and prognosis. On one side, the presence of bilateral hydronephrosis with or without the keyhole sign in a male fetus should always raise the suspicion of posterior urethral valves. One the other side, different indicators can be suggestive for this pathology, including an enlarged fetal bladder $(>7 \mathrm{~mm})$ in the first trimester and poor bladder cycling $[2,3,4]$.

Besides the diagnostic criteria, several elements in the prenatal evaluation have a prognostic role, allowing the clinician to make predictions regarding the short- and longterm outcome. Progressive oligohydramnios and anhydramnios are associated with a high risk of severe lung dysplasia and death. Furthermore, a poor echogenity of the renal cortex and a poor bladder filling during the ultrasonography are signs of poor prognosis and are associated with renal failure in newborns [5]. According to Sarhan et al 2017, the birth weight does not influence the prognosis regarding kidney function in patients with PUV. However, newborns with low birth weight have a higher risk for vesicostomy, longer hospitalization, vesicoureteral reflux and higher nadir creatinine [6, 7].

Regarding the antenatal evaluation of patients with PUV, an important mention has to be made with regard to the in utero therapeutic options. Fetoscopy, decompression of the bladder by vesicoamniotic shunting or fetal cystoscopy with valve ablation are among the considered options. Nevertheless, the randomized controlled PLUTO trial and Nassr meta-analysis in 2017 showed no long-term benefits on renal function $[8,9]$.

\section{Sequelae of PUV}

The bladder outlet obstruction during the fetal development creates a series of consequences, that affect the patients with PUV, despite proper and early treatment of the valves. The bladder dysfunction is progressive, from a hypertrophied bladder with good voiding in the compensated phase, to a decompensated bladder, without adequate emptying [2,3].

Two major events take place during the compensated phase. First of all, the bladder hypertrophy corelates with high voiding pressures, which further lead to the remodeling of the bladder wall. Secondly, the co-existing renal dysplasia causes polyuria, which in turn increases the urinary volume that needs to be stored by the bladder. The dilatation of the bladder represents the beginning of the decompensated phase and places the upper tract at risk for higher dilatation [2].

Ureteral dilatation is correlated with the vesicoureteral reflux, which is seen in 70 per cent of patients with PUV, but it can also be determined by the increased bladder pressure and by the polyuria from the renal dysplasia. The chronic ureteral dilatation leads to ureteral wall thickening, loss of peristalsis, urine stasis and increased pressure in the renal pelvis [3]. The increased pelvic pressure produces alterations in the morphology and function of the kidneys, leading to irreversible dysplastic changes and poorly concentrated urine.

\section{Biologic markers}

As part of the paraclinical evaluation for patients with PUV, serum creatinine level was initially considered an important prognosis marker. However, it has failed to prove its accuracy over time, and, during the last two decades, it has been replaced with nadir creatinine. This represents the lowest creatinine level during the first year after diagnosis and shows a strong correlation with long term risk of CKD and ESKD. Coleman et al. divided the patient into risk groups, based on the value of nadir creatinine. A low risk is represented by values of nadir creatinine lower than 35 $\mu \mathrm{mol} / \mathrm{L}$ or $40 \mathrm{mg} / \mathrm{dL}$ and the high-risk group includes the patients with nadir creatinine higher than 75 $\mu \mathrm{mol} / \mathrm{L}(0.85 \mathrm{mg} / \mathrm{dl})$. Another parameter, introduced more recently, is creatinine velocity, defined as the change in the level of creatinine after the urinary drainage. An increase more than $3 \mu \mathrm{mol} / \mathrm{L} /$ day is associated with a higher risk of progression of the disease towards CKD. Coleman also affirms that the association of the creatinine velocity $>3 \mathrm{mmol} / \mathrm{L} /$ day in patients from the high-risk group - based on nadir creatinine level - will lead to CKD in 100 per cent of the cases. In the same time, the patients from the low risk group, with nadir creatinine $<35 \mu \mathrm{mol} / \mathrm{L}$ and creatinine velocity $<3 \mu \mathrm{mol} / \mathrm{L} /$ day are at very low risk of CKD [10, 11].

The urinary cytokines TGF-1, TNF- $\alpha$, IL-6 and microalbuminuria are mentioned in a lot of studies, but their predictive value for long term renal function still needs to be proven. Mandelia describes a raise in the postoperative values of these biomarkers, compared to the preoperative values, in patients with risk of long-term renal damage, a decrease of the postoperative values being associated with a good outcome [12]. Three other studies analyse the prognostic value of the urinary inflammatory cytokines in obstructive nephropathies, showing that increased values may be correlated to progressive renal insufficiency $[13,14,15]$. In one study, Vieira suggested that the inflammatory molecules have a high level in fetuses with PUV at 22 weeks of gestation, showing an important role of inflammation right after the valve formation [15]. The plasmatic renin activity is proposed as an early prognostic factor by Bajpai, but further investigations are needed in order to clarify the mechanisms involved [16].

\section{Imagistic factors}

In 2016, Odeh presented the results of a study involving ultrasonographic kidney measurements at patient's admission and the correlation with the clinical outcome. The conclusion of his study is that estimated renal parenchyma, the cortico-medullar index and the renal echogenity may represent prediction factors for the longterm outcome [17].

Another imagistic index was suggested by Niyogi, who proposed the acronym SWRD (shape, wall, reflux, diverticula), representing the cystographic measurements of 
the patients with PUV. According to him, a high SWRD index describes a bladder dysfunction with low compliance and high pressure. The study also presents the correlation between a high index and the need for an invasive method of urinary drainage - vesicostomy, urinary derivation [18].

\section{$\underline{\text { Urodynamic studies }}$}

Urodynamics is an essential part of the evaluation of children with PUV, allowing an estimation of the bladder contractility. The progression of bladder dysfunction represents an independent factor that worsens the prognostic. Several theories try to explain the mechanism underlying bladder hypocontractility and myogenic failure, including the hyperactivity of the detrusor muscle, the bladder neck hypertrophy, the co-existent vesicoureteral reflux and the anticholinergic medication. The morphologic changes are represented by the increasing extracellular matrix in the bladder wall, as well as changing the ratio between collagen type III and I [19, 20].

In a recent study, Ansari analyzed urodynamic parameters and their correlation with chronic kidney disease, showing a strong association between the bladder contractility index, calculated with the formula $\mathrm{BCI}=$ PdetQmax+5Qmax, and the long-term progression towards chronic kidney disease stage III [20].

\section{Urological management of bladder dysfunction}

Knowing the type of bladder dysfunction in patients with PUV plays a vital role in order to establish a proper management and to reduce the progression to end stage kidney disease, but also to maintain a bladder function that is suitable for a renal transplantation.

The pharmacological treatment plays an important role in the treatment of bladder dysfunction. Anticholinergic medication (oxybutynin, solifenacin) are useful for low- capacity bladder, with high pressure, and for hypercontractile bladder. The high-pressure bladder can also be managed medically, with tricyclic antidepressants (imipramine). Alpha blockers (terazosin, tamsulosin) are used for bladder neck hypertrophy with outlet obstruction or bladder neck obstruction and myogenic failure. Incomplete bladder emptying with clinical sequelae despite medication can be managed by clean intermittent catheterization.

Regarding renal transplantation, poor graft survival was initially reported in patients with PUV, probably because of a persistent bladder dysfunction. Before the renal transplant, the bladder should store and empty at low pressures [3]. An augmentation cystoplasty is recommended before transplant, in the case of a poorly managed bladder dysfunction. In their recent study, Saad et al. idetified no difference in long term graft outcome in patients with or without lower urinary tract disorders, in the presence of a good bladder emptying [21].

\section{Conclusion}

Posterior urethral valves represent a complex malformation, that involves the whole urinary tract. Unfortunately, the treatment of the urethral obstruction does not bring definitive cure for the patients. Except the nadir creatinine and creatinine velocity, the biologic markers still need to prove their predictive value for CKD. Imagistic measurements, such as ultrasonographic kidney measurements, cystographic SWRD index, as well as the bladder contractility index determined by urodynamic studies, constitute a key element of diagnostic. Altogether, these factors play an essential role in creating an estimation of risk for children with PUV. This stratification, correlated with a proper management of bladder dysfunction, allow the maintenance of good bladder function and the prevention or delay of the evolution towards renal failure.

\section{Conflict of interest: NO CONFLICT}

\section{References}

1. Bilgutay, A.N., Roth, D.R., Gonzales, E.T.Jr., Janzen, N., Zhang, W., Koh, C.J., et al. Posterior urethral valves: Risk factors for progression to renal failure. Journal of Pediatric Urology. 2016;12(3):179.e1-7.

2. Wein, A.J., Kavoussi, L. R., \& Campbell, M. F. (2012). Campbell-Walsh urology (10th ed.). Philadelphia: Elsevier Saunders, pp. 565-567.

3. Deshpande, A.V. Current strategies to predict and manage sequelae of posterior urethral valves in children. Pediatric Nephrology. 2018 Oct;33(10):16511661.

4. Chevalier, R.L. Prognostic factors and biomarkers of congenital obstructive nephropathy. Pediatric Nephrology. 2016;31(9):1411-20.

5. Harper, L., Waubant, A., Vignes, J., Amat, S., Dobremez, E., et al. Can quantity of amniotic fluid reliably predict postnatal renal function in boys with posterior urethral valves: a decision curve analysis. Prenatal Diagnosis. 2017;37(9):931-4.

6. Sarhan, O.M. Posterior urethral valves: Impact of low birth weight and preterm delivery on the final renal outcome. Arab Journal of Urology. 2017;15(2):159-65.

7. DeFoor, W.R.Jr. Challenges in Predicting Renal Outcomes in Boys with Posterior Urethral Valves. The Journal of Urology. 2016;196(3):639-40.

8. Nassr, A.A,, Shazly, S.A.M., Abdelmagied, A.M., Araujo, E.Jr., Tonni, G., et al. Effectiveness of vesicoamniotic shunt in fetuses with congenital lower urinary tract obstruction: an updated systematic review and meta-analysis. Ultrasound Obstet Gynecol. 2017 Jun;49(6):696-703 


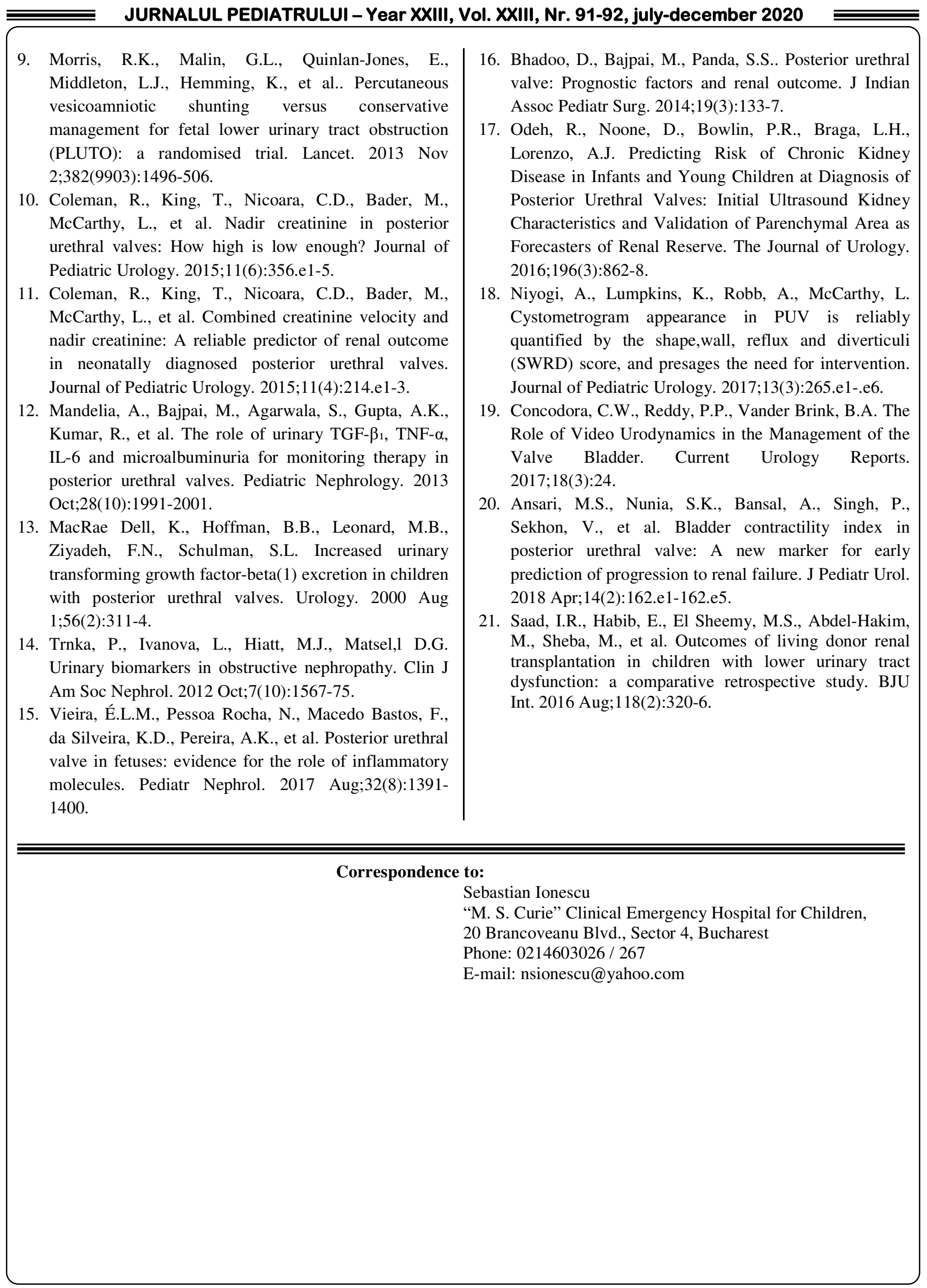

